\title{
Situation Awareness Data Fusion Method Based on Library Events
}

\author{
Haixu Xi ${ }^{1,2}$, Wei Gao ${ }^{2, *}$ and Gyun Yeol Park ${ }^{3}$ \\ ${ }^{1}$ School of Economics and Management, Nanjing University of Science and Technology, Nanjing, 210094, Jiangsu, China \\ ${ }^{2}$ School of Computer Engineering, Jiangsu University of Technology, Changzhou, 213001, China \\ ${ }^{3}$ Gyeongsang National University, Jinju, 52828, Korea \\ ${ }^{*}$ Corresponding Author: Wei Gao. Email: gw@jsut.edu.cn \\ Received: 26 July 2021; Accepted: 22 September 2021
}

\begin{abstract}
Microelectronic technology and communication technology are developed in deep manner; the computing mode has been transferred from traditional computer-centered to human centered pervasive. So, the concept of Internet of things (IoT) is gradually put forward, which allows people to access information about their surroundings on demand through different terminals. The library is the major public space for human to read and learn. How to provide a more comfortable library environment to better meet people's learning requirements is a place where the Internet of things plays its role. The purpose of this paper is to solve the difference between the data fusion of library environment and the data fusion of other environments by the method of data fusion oriented to library. This paper presents a general technical framework of situational awareness for smart library system which includes a data fusion middleware. It can process data and inform the upper module of the changed library environment after deploying the smart library system in a library, including data collection and processing, how to judge whether events are triggered, how the system reacts, and the acquisition and update of user preferences. This paper presents a situational awareness recommendation method based on an effective data fusion model and algorithm for library after conducting experimental in service of library, which give more accurate of book recommendation than traditional method and good learning service environment of library for readers.
\end{abstract}

Keywords: Intelligent library; internet of things; situational awareness; information fusion; data fusion

\section{Introduction}

With the emergence and development of various new technologies, people also put forward higher demands for improving their quality of life. The library, which occupies the space of people's life, has also entered the list of human beings to transform it [1]. The emergence of a new computing model pervasive computing provides a solution to this problem. It emphasizes computing integrated with the environment, and people can obtain and process information at anytime, anywhere and in any way [2-4]. When applied to library, such anytime, anywhere interaction mode requires that the devices arranged in

This work is licensed under a Creative Commons Attribution 4.0 International License, which permits unrestricted use, distribution, and reproduction in any medium, provided the original work is properly cited. 
the library can perceive the changes in the environment of the library, and then make automatic behaviors based on user needs or pre-set according to the changes in the environment. If you want to perceive the change of environment in the library, it is inseparable from the perception of the network, the sensor networks can be made up of cable equipment, can also be composed of a wireless device, its task is to pass the sense data of all kinds of physical devices in the network to obtain the data changes of library environment, the data through the preliminary processing will be sent to the upper module for use [5-7].

The Internet of things is a data network and control network composed of a variety of intelligent devices. Intelligent devices on the Internet of things of Library can perceive the external environment and obtain multi-dimensional data. Through the analysis of these data, decisions can be made manually or automatically, and control instructions can be issued to control the operation of intelligent equipment, so as to realize the function of intelligent library, meet the above humanized and intelligent pervasive computing requirements, and improve user satisfaction. In different library services, it is necessary to integrate different sensing information in Internet of things equipment for analysis, to provide more suitable service contents and methods. Therefore, the data fusion analysis of sensor data has become the key to the realization of library services based on Internet of things.

The first part of this paper introduces the background significance and related work. The second section puts forward the technical framework of the intelligent library system deployed in a library, which provides some references for the standardized design system. The third section, aiming at the special environment of library, puts forward the characteristics of data fusion in this environment and the methods and models to be adopted. The fourth section mainly introduces the design and implementation of intelligent library data fusion middleware. The fifth section is the conclusion.

\section{Related Works}

\subsection{Related Technical of Intelligence Library Construction}

Gaohui Cao aims to define the concept of intelligent library and put forward an overall method of constructing intelligent library by combining the current practice and the latest technology. Based on an extensive review of existing library construction documents and practices, this paper makes a distinction between similar types of intelligent libraries and divides the relevant concepts of intelligent library construction into three dimensions: technology, service and people. Traditional libraries can transform to intelligent libraries through the strategic design and implementation of cloud computing, data mining, artificial intelligence, and other advanced technologies, but they also need to consider service construction, user training and librarian training. Aiming at the three dimensions of intelligent library (technology, service and humanities), this study clarified the concept of intelligent library and proposed strategies [8,9]. As a new mode of library, Intelligent library's green development requires providing convenience for readers and protecting their privacy at the same time. Wang Jialing analyzed privacy risks brought by digital technology, network technology, and data mining technology from two perspectives of network platform and physical library. Then, combined with the existing information security technology and network security technology, the corresponding technology protection strategy is proposed [10].

The extraction of visual information is essential for controlling movement in dangerous situations and maintaining situational awareness. The possibility of injury or death will be greatly improved when changing coordination with wearing protective gear. Jongil Lim examined the effects of load size and distribution of environmental perception, coordination arrangement of segmental, and the type of head gaze. Experiment shows that when some soldiers stepped down from high floor and identify visual information with the protective gear, additional packaging and helmet loads extends the visual information recognition time, and small increase in helmet loads impact higher. Under lighter loads, the head-guidance and the same 
phase of the trunk-head coordination is stronger, while under heavier loads, the trunk head coordination is enhanced and the head-gaze dynamics is more disturbed [11-13].

\subsection{Research Status of Situational Awareness}

Computer network is developed, and people are no longer satisfied with the interconnection between people but want to be able to connect between objects and between people and objects. Therefore, the concept of Internet of things is proposed, and it has been widely used in manufacturing, military applications, transportation, aerospace, and other fields.

The Internet of things comes from pervasive computing, which was proposed in 1991. It is generally believed that pervasive computing should be that computers exist anywhere in the physical world, but users do not know that these computers are providing them with various services [14,15]. Then in 1994, situational awareness was defined as a computer system with adaptive responses to the surrounding environment, illustrating the importance of the surrounding environment. Former researchers used examples of situations to define situations. It is defined as follows: according to the user task, the system uses situational information to provide relevant information or services to the user. From this definition, the goal of situational awareness is the same as that of the personalized system. they all provide information and services based on the user's situation and personal interests separately. So, according to Endsley's definition, situation awareness can let user knowing what is going on around you [16-18]. As a form of computing service, situational awareness computing is adaptable, responsible and has the characteristics of emplacement and environmental orientation. At present, the application of situational awareness mainly focuses on smart space, such as collecting the user's location information, automatically transferring the phone for the user, opening the $\mathrm{X}$ Window session for the user, reminding the user what to do, assisting the user to interact with nearby objects, recommending media information to the user, and taking care of the elderly [19-21].

Situational awareness is widely used not only in the field of pervasive computing, but also in the spatial service of Smart Library. Through the upgrading and transformation of the smart library space, situational awareness service can provide a variety of experiential services such as learning, exhibition, education and teaching, online and offline services, and actively improve the service efficiency of the smart library space service, the coverage of reference services, and the accuracy and timeliness of indoor positioning and navigation, Enhance users' on-the-spot experience in the smart library space; The smart library context awareness service can provide users with targeted push services based on user context by tracking and analyzing users' behavior in the library, combined with users' time context and historical behavior context. Therefore, the smart library context awareness application can better realize personalized services in combination with users' real-time needs; Smart library uses mobile network environment and Internet of things technology to enable users to experience the effective integration of online and offline services when they are in the physical library environment. The smart library service combined with situational perception has the characteristics of universality, initiative, accuracy and flexibility.

\section{Methodology}

\subsection{Basic Structure of Intelligent Library}

It shows the intelligent library system in this system has a variety of physical equipment and communication methods, which mainly includes two network parts, one is the Internet of things devices deployed in the library, the other is through the Wide area network (WAN) and user dialogue terminal. All the data collected from the device are considered context awareness which can confirmed whether the service is good for readers. They can be broadly divided into three parts: the Internet of things part inside the library which mainly collects real-time environmental information of library; The wisdom box 
section, which is responsible for judging environmental changes and responding to library members and events. The user dialogue terminals include service centers outside the library and users' personal mobile devices [22-24].

\subsection{System Architecture of Wisdom Magic Box System}

The wisdom box is responsible for further processing of data from the Internet of things and needs to respond to changes in the library environment. It also serves to separate the underlying hardware device from the upper service module. The wisdom magic box consists of server and man-machine interface. The whole intelligent library system is deployed in the server part of the wisdom magic box. Middleware is in the position of the whole magic box and its indispensable role. It is mainly composed of two blocks: the first is the library service network, whose function is to provide services to library members through existing local programs for events caused by changes in the library environment, or to transmit a service request to a remote service center; The other is the Internet of things, which requires the management of hardware devices deployed in the library, including the management of different communication protocols, registration and deletion of different devices [25-27].

The intelligent library system is deployed in the wisdom magic box, which is divided into six layers: Internet of things layer, data layer, event layer, situation layer, pattern layer and service layer. Data is transferred in different semantics between different layers, which cover the device interface, that is, the common interface to physical devices. Data filtering, data transformation, data classification and other data level fusion of the data received by the Internet of things are handled in data layer [28-30]. After obtaining accurate, stable, semantic data, according to the rules to combine the data into a specific or some events in event layer. In the context layer, the single event, or multiple events to be obtained can be converted into one or some situations according to the event-to-situation mapping rules. Pattern layer, in which a large number of specific situation combinations are stored, reflects the specific state of the library in a macro sense [31-33]. For example, when a guest enters the library, the system can automatically launch the corresponding service. The service layer, also known as the application layer, directly interacts with the user, communicates with people in a friendly way, and will directly provide corresponding services to the owner. The overall system architecture of wisdom magic box system can be concluded as five layers:

\section{(1) Device interface}

Haier interface, provided by Haier, is used to receive and send information from Haier equipment. We can call the interface directly. Wireless sensor network (WSN) interface, which is composed of other wireless or wired sensors and devices in the library, most of which adopt ZigBee communication protocol. 5G technology is a new communication technology produced with the development of mobile phones. It can provide users with rich multimedia information, reaching $144 \mathrm{~kb} / \mathrm{s}$ when the device is moving at high speed, $384 \mathrm{~kb} / \mathrm{s}$ when walking, and $2 \mathrm{Mb} / \mathrm{s}$ when at rest [34,35].

\section{(2) Data layer}

The data value is converted in this layer, and the converted data is further processed. Various denoising methods, such as grouping average and moving average, are applied to ensure that the obtained data reflects the changes of the library environment most truly. User preferences are also merged on this layer and updated to prepare for the next event fusion.

Covariance cross fusion algorithm is an algorithm that combines the estimated values of each sensor when the cross covariance of each sensor is unknown in Kalman filter. For example, to fuse the measured values of two sensors $A$ and $B$ with the same property, fuse the average/covariance $C$ of the estimated value $C$. The calculation formula of is shown in (1), where $A$ and $B$ are the mean/covariance of $A$ and $B$, $\omega$ is the adjustment coefficient. 
$\hat{c}=C\left(\omega A^{-1} \hat{a}+(1-\omega) B^{-1} \hat{b}\right)$

\section{(3) Event layer}

After data conversion and filtering, the event layer follows event inference rules, and matches the data obtained from the data layer into corresponding events based on user preferences. Here we use the production rule system for reasoning.

\section{(4) Database layer}

The data in the database can be divided into two kinds. One is static data, that is, all kinds of data that have been input before the program runs, such as the location information of each sensor in the home, personal data of family members and personal preferences of each person. The local database stores this data in the form of a data table. The other is dynamic data, that is, real-time information collected through the underlying Internet of things during the operation of the intelligent library system. This data is either dynamically queried by Tiny DB from a sensor node or from other types of sensor devices.

(5) Rule base layer

The rules in the rule library are defined when the system is initialized, but it also adds new rules during system use to meet the needs of the system. Rules are stored in the database when they are defined and passed into the matching function as an input when they are matched.

\subsection{Middleware Architecture}

In the Joint directors of laboratories (JDL) model, the process of data fusion is described as follows. First, data from different sensors, database, priori information, human interaction or other source are filtered and classified or signature. Second, in the object refinement level, the coordinates and units of data are unified and classified into position, motion and other data types, and are associated with objects, as well as finely counted and estimated. Third, in the situation refinement level, the contextual description of relation between the objects and observed events are defined. Forth, in the threat refinement level, inferences about future situation or danger are conducted for the solution and countermeasures. Fifth, the process should be optimized after monitoring the performance of the system, such as reallocates sensors or real-time constraints. Sixth, the intelligent application can be introduced based on the analyzing of data, such as personalized knowledge recommendation.

The middleware of the intelligent library system is used to process a variety of data collected by the underlying Internet of things and transform them into data form that can be used by the upper module. This middleware provide top event interface directly, after the sensors perceive a certain trend in the library, it will immediately send the data to data fusion middleware, after the events of the middleware fusion center, according to the user's personal habits formed after it put some relevant data for processing an event, the event is sent into the event interface, this event will be placed in the upper module layer after receiving the user's instructions, situational layer will put these instructions into the corresponding situation events, these events should be handled to find out the corresponding sensors through data integration middleware, these events and the middleware sent the corresponding information to their corresponding sensor driver module, and then the Internet layer of sensor will perform some operations, to provide users with services you need. The middleware also needs to give these events a unified definition format, such as the main body of the event to describe what the object is, which person, which thing; What is the state of the person or thing, such as its position, where it is moving, what is the temperature, what is the heartbeat, when data of time was collected, whether it was urgent data can be decided which can indicate its processing priority, and so on. 
The architecture of the data fusion middleware is shown in Fig. 1. It mainly deals with two major data streams: the first is the data transferred from the hardware deployed in the library to reflect the changes of the physical environment in the library in real time. The middleware should conduct preliminary processing and fusion of these data, and then combine relevant data into one or some events that can describe the current situation of the library, and then pass the event to the context layer to match a certain situation. Another data flow is from the upper module's instructions, they mainly according to the current user or system needs to execute a query, a state or the hardware setup, let the hardware to complete certain operations. These instructions require service optimization, the decomposition of the final instance into a command for specific hardware devices, into these commands parsing running corresponding hardware and then the hardware can execute the command.

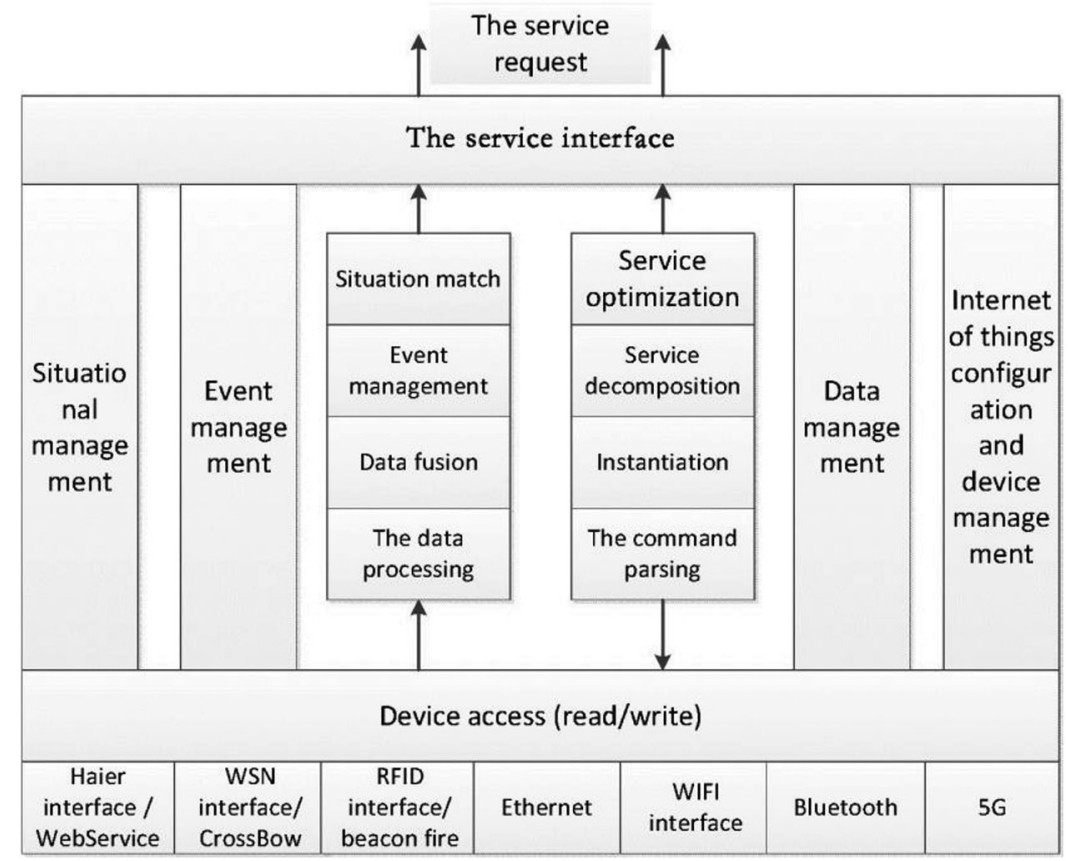

Figure 1: Middleware architecture

\section{Experiments}

According to the above-mentioned technical architecture of Internet of things of Library, this study has built a library intelligent service system. The system provides readers with library services such as book borrowing and learning environment by collecting information such as ambient temperature, light and user access records.

From the Internet of things to the real-time information of the library environment to the final response of the system, the whole process requires three steps: first, data collection and processing; second, determine whether an event is triggered at this time; finally, the system responds to the event.

\subsection{Data Collection}

According to the source of information, the sensors can be divided into the following parts:

(1) Infrared sensors: these sensors are mainly responsible for position detection, which are used to detect the target distance, determine the target position, and determine the target state, category and whether there is any human activity in a certain space. 
(2) Access control, alarm, glass breakage sensor, and monitor: when such sensors are triggered, they will transmit information to the upper layer of middleware and send a sound. The sound alarms the indoor owner, and its internal information will cooperate with other sensors to determine the source of the alarm.

(3) Light intensity sensor, water sensor, temperature sensor, pressure sensor, smoke sensor, Co and $\mathrm{CH} 4$ gas sensor: this kind of sensor is used to sense physical phenomena in the library, according to the acquired information, the system will do the corresponding operation to adjust people surrounding environment to make people's lives more comfortable.

(4) Intelligent switch, leakage protector, standby power supply: this kind of equipment is controlled by the upper instructions and is responsible for the control of power supply in the library and the treatment of power failure.

(5) Intelligent bookshelf, intelligent air conditioner, intelligent desk, intelligent stereo, intelligent security check, intelligent meter and intelligent exhaust fan: this kind of information provides the information of various household appliances to the intelligent library system and is controlled by the upper instructions. Equipment includes all smart appliances in the library.

(6) Communication network and the Internet: it is wisdom library system one of the important source of information in the information fusion system, intelligent library system through the Internet and communicate information to the master and communication network system, and through it to get information from outside the library or the master's instructions, but also can get some real time information from the Internet to provide service for the family.

(7) System time: As important reference information, time determines whether many events should be triggered, such as most events related to turning on lights.

In this experiment, the Smart Library of Jiangsu University of Technology is selected as the research object. Each floor of the library is equipped with wisdom magic box system to process the temperature and humidity of each layer and the document status information transmitted from the smart bookshelf. Sensor data is sensed unidirectionally by system equipment, non-interactive sensors, and software. Its acquisition method mainly identifies and senses the target object through the equipment and software system, and then realizes the perception and acquisition of data through its own application settings. In order to verify the effect of the Smart Library Service proposed in this paper, 10 research teachers from different disciplines and 52 undergraduates from different colleges and universities were selected to borrow literature in the smart library, leave the process records of scientific research or learning, and accept the user satisfaction survey of smart library services.

\subsection{Data Processing}

The processing of the data in this system is divided into four steps:

(1) Convert the obtained data into data types

(2) Integrate the data of a single sensor

(3) Processing of sensors in a specific area (accuracy can be increased by grouping geographical features)

(4) Analyze the trend reflected by the sensor data in this specific area

\subsection{Data Value Conversion}

When the system gets the data, these data are just a series of Numbers, such as $228,779,1199$, which cannot be recognized by people, let alone facilitate people to understand the physical meaning they 
represent. Therefore, according to the physical characteristics of the equipment and some indicators, this paper lists several data value conversion formulas.

Using this formula, the system converts the collected data into the data can be identified and have physical semantics, including temperature, light intensity, and voltage. The constants' values of formulas are all the experimental value of the equipment's manufacturer. These formulas are defined as follows.

(1) Temperature conversion formula

$T=\frac{1.0}{a+b * \operatorname{In}(r t h r)+c *(\operatorname{In}(r t h r))^{5}}-273$

where $a, b$ and $c$ are constant coefficients, $a=0.001307050, \mathrm{~b}=0.000214384, \mathrm{c}=0.000000093, \mathrm{x}$ is the real-time temperature data collected by the system. $r$ th $r=\frac{10000 *(1023-x)}{x}$.

(2) Light intensity conversion formula

$L=\operatorname{light} *\left(\frac{a}{\text { voltage }}\right) / b$

$a$ and $b$ are constants, $a=1252352$ and $\mathrm{b}=1023$. Light is the real-time data of the light intensity obtained by the system, and voltage is the real-time voltage data obtained by the system.

(3) Voltage conversion formula

$v=\frac{a}{x}$

A is a constant, $a=1252352$, and $x$ is the real-time data value obtained by the system.

After the data conversion, the status of environment can be determined in real time. For example, when the $\mathrm{T}$ is $40^{\circ} \mathrm{C}$ and the light intensity are high, and the voltage is low, and we can infer that library is not suit for readers at evening.

\subsection{Matching and Execution Process of Experimental Rules}

The trigger of the event must satisfy two the following conditions. 1). the atomic event that constitutes the complex event has occurred; 2). These atomic events have occurred in time.

According to the JDL model, when the conditions for defining an event are met, the event is triggered, and the inference method of the production system is adopted here. Each condition is expressed by production knowledge representation. The general form of production is $\langle$ predecessor $\rangle \rightarrow\langle$ postdecessor $\rangle$, where the former is a condition and the latter is the result derived. The antecedents are composed of data expressions and connectors. However, in this system, the former is composed of atomic events, and the latter is the events derived according to the conditions. The production system completes the work process by pattern matching, conflict resolution and rule execution, as shown in Fig. 2.

(1) Pattern matching: Starting from the first rule in the knowledge base, scan all rules in the rule base one by one, compare all elements in the dynamic database with the precursors of each rule, so as to find all rules that meet the conditions. If all conditions in a rule preceding a rule in a rule library match the current fact state in the dynamic database, the rule is added to the conflict set, and the following rules are checked until all rules in the rule library have been matched.

(2) Conflict resolution: Conflict refers to the state in which multiple rules are successfully matched at the same time. At this point, it is necessary to choose which rule should be triggered according to the pre-set evaluation criteria. There are many ways to resolve the conflict, such as choosing according to the order 
of successful match, that is, choosing the rule that succeeds in the first match; Select according to priority, that is, set the magnitude of each event and the rule with the highest priority option value in the conflict; According to the order of execution, the rule of the last execution is selected first when the conflict occurs; According to the degree of detail selection, that is, in the case of conflict, priority is given to the rule that the premise part includes the maximum number of sub-conditions; According to whether the rule has used the selection, choose the rules that have not been used before; Select by new facts, that is, in the event of a conflict, give preference to rules related to the latest facts added to the dynamic database. In this system, the method selected according to the order of successful matching is adopted, because the sensor network requires the system to make a quick response, while other methods will take up a lot of computer time.

(3) The reasoning of the generative system can be divided into three basic modes: Forward reasoning, backward reasoning and mixed reasoning. Forward reasoning, which starts from a set of conditions representing facts and uses some rules of reasoning to prove whether the objective conclusion is true or not; Backward reasoning, which starts from the conclusion representing the target, uses certain rules to prove the fact condition is true, that is, gives a variety of hypothesis (target) situations, and then verifies whether these hypotheses are true one by one; Mixed reasoning takes the advantage of the first two methods, which proceed from both conditions and objectives, and if the two parties meet somewhere in the middle, the reasoning succeeds.

In this system, forward reasoning is adopted, because its goal is to find all possible conclusions from a set of given facts, which is in line with the actual situation of the system, deducing possible results from the occurrence of atomic events, and this way is more in line with people's logic.

(4) Rule execution. Running the reasoning mechanism of repeated cycle to perform the above three stages, it is run according to the facts and rules of the system in dynamic database in the library knowledge, constantly introduced the unknown by the known facts to dynamic database once again. The reasoning process of transforming them into a new premise or fact cycle until the conclusion is deduced.

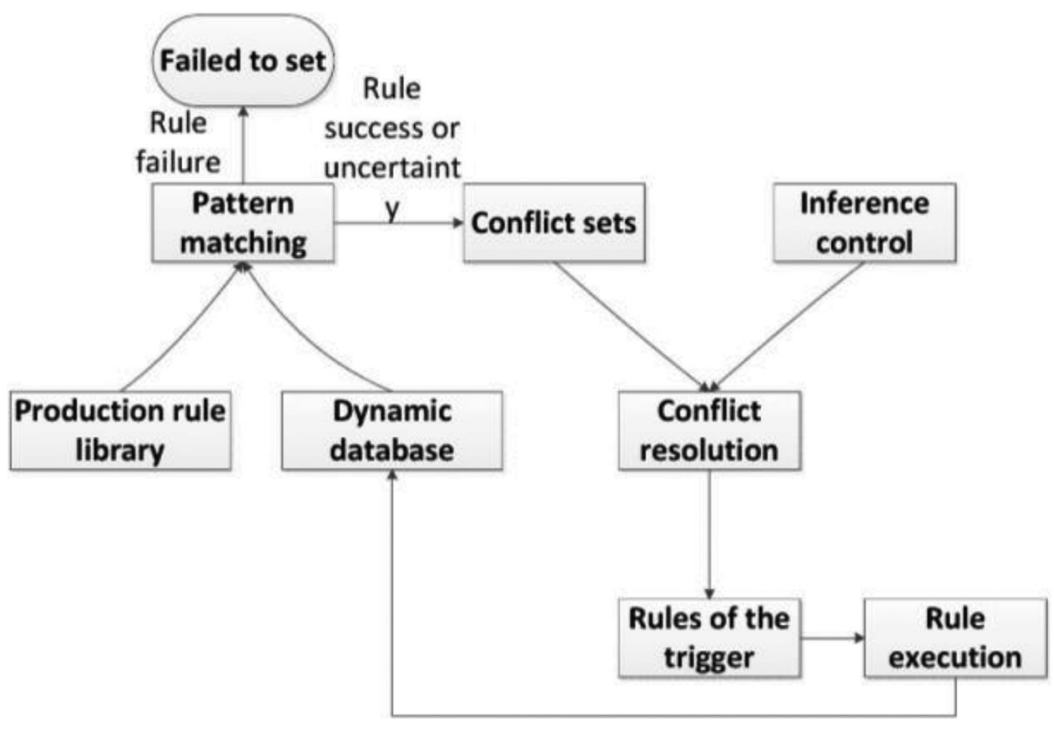

Figure 2: Working process of production system 


\section{Results and Discussion}

\subsection{Analysis of Situational Awareness}

Collaborative filtering method provides personalized literature resources for readers, and it is the main content of smart library service. Collaborative filtering finds users' preference for literature topics through historical data of users' reading literature resources, divides users or articles into groups based on different preferences, and recommends to readers the documents read by similar readers or similar literatures that the readers have read. These are the collaborative filtering algorithms based on items and articles in traditional collaborative filtering algorithm. The situational awareness collaborative filtering algorithm considers the context information in recommendation. In order to verify the effectiveness of the situational awareness collaborative filtering method proposed, we defined a new evaluation indicator from the perspective of reader experience: Reader acceptance ratio (RAR), calculated as follows:

$R A R=\frac{\left.\sum_{\text {InterestSet }}\left\{I_{1}, I_{2} \ldots I_{m}\right\} I \text { Top }-N \text { Document }\right\}}{\{\text { Top }-N \text { Document }\}}$

where in formulas (5), $\{$ Top - NDocument $\}$ represents the literature resources of the Top- $n$ ranked according to the recommended method, and the interetrate set $\mathrm{I}_{1}, \mathrm{I}_{2} \ldots \mathrm{Im}$ represents the literature resources of interest to the target reader. Find the intersection of the two sets, representing the resources of interest to the reader in the list of recommended resources. From the perspective of reader experience, reader acceptance reflects the degree of resource recommendation adoption. The higher the value, the more likely the recommendation method is to recommend appropriate resources to target readers in the current context.

There are two types of data used in the situational awareness collaborative filtering method, one is the data input by readers in their daily activity in library service, and another is the data collected by the distributed sensor of internet of things in the library. Conditional entropy is used to calculate the influence value of each situational attribute in the recommendation process, distinguish the situational attributes with different influence, and measure the weight of each situational attribute in the recommendation process according to the situational conditional entropy.

Situational entropy is the uncertainty of readers' choice of items on the premise of knowing a certain situational attribute. The greater the conditional entropy, the smaller the influence of the context attribute on readers' choice of resource types; The smaller the conditional entropy, the greater the influence of the context attribute on readers' choice of resource types. The definition of situational conditional entropy is as follows:

$H(I \mid c)=\sum_{i=1}^{m} P\left(C_{i}\right) \sum_{j=1}^{n} P\left(I_{j} \mid C_{i}\right) \log _{2} P\left(I_{j} \mid C_{i}\right)$

where, each situational attribute $c$ has $\mathrm{m}$ situational values, and $P\left(C_{i}\right)$ is the probability that the situational attribute in the situational factors is $C_{i} ; P\left(I_{j} \mid C_{i}\right)$ indicates the probability that readers choose $I_{j}$ resources under the condition that the situational attribute is $C_{i}$.

The target reader is A. Suppose that the item set jointly evaluated by readers A and B in the same context is $\mathrm{I}_{\mathrm{ab}}$, and use Pearson Correlation Coefficient to measure the similarity between readers A and B in a specific context, then the similarity between readers $\mathrm{A}$ and $\mathrm{B}$ is:

$$
\operatorname{sim}(a, b)=\frac{\sum_{i \in I_{a b}}\left(R_{a, i, c}-\overline{\overline{R_{a, c}}}\right)\left(R_{b, i, c}-\overline{\overline{R_{b, c}}}\right)}{\sqrt{\sum_{i \in I_{a b}}\left(R_{a, i, c}-\overline{\overline{R_{a, c}}}\right)^{2} \sum_{i \in I_{a b}}\left(R_{b, i, c}-\overline{\overline{R_{b, c}}}\right)^{2}}}
$$


According to the acceptability of readers, the situational awareness collaborative filtering method is compared with the traditional method. We use k-folding cross test to conduct data modeling and testing. Firstly, the dataset is divided into K parts, among which k-1 part is used as training data set and the other 1 part is used as test data set. At the same time, the cross-examination process is repeated many times to make the experimental results more reliable and objective. In the experiment, we applied k-fold cross test $(\mathrm{K}=10)$ to repeat the experiment for 20 times and recommended the literature resources ranked Top 5 to the target readers. The comparison results of the two collaborative filtering recommendation methods on the reader acceptance index are shown in Fig. 3.

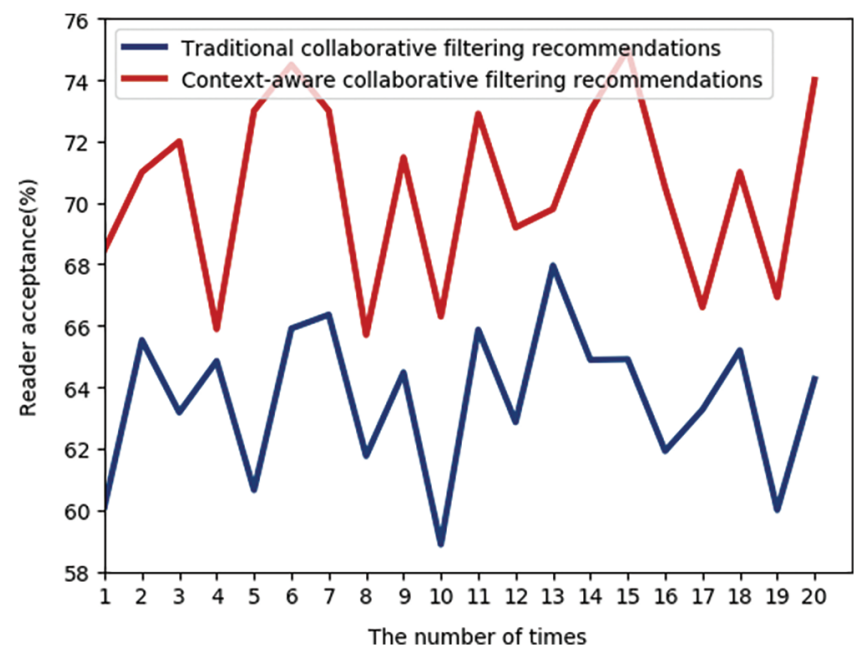

Figure 3: Comparison of two collaborative filtering information recommendation methods

As can be seen from Fig. 3, the context-aware collaborative filtering recommendation method proposed by us is better than the traditional recommendation method based on collaborative filtering in performance in terms of reader acceptance. The difference is about $10 \%$. The experimental results show that the collaborative filtering recommendation method based on situation awareness, can well predict the reader perception properties on the situation, and identify the different role in the different situation for information recommendation, which based on the current situation in mobile environment information, accurately provide personalized mobile reading service for readers. Readers can get more satisfactory literature resources recommendation in different light, different time periods and different library areas. Compared with the traditional collaborative recommendation, the value of RAR is higher, up to 75 and the F-Measure value is 2.415 .

\subsection{Book Recommendation Based on Situational Awareness}

A collaborative filtering instance of context awareness based on book similarity are shown in Tab. 1. User A borrows Book 1 and Book 2. The indicator is an evaluation indicator calculated based on the location of the book and the suitable time period. Book $A^{*}$, Book $B^{*}$ and book $C^{*}$ are the product weights of the similarity of commodity $A, B, C$ with book 1 and book 2 and the evaluation indicator respectively. The total/indicator calculated is sorted in descending order and recommended to user $A$. 
Table 1: Example of recommendation for user A

\begin{tabular}{|c|c|c|c|c|c|c|c|}
\hline & Indicator & Book $A$ & Book $A^{*}$ & Book $B$ & Book $B^{*}$ & Book $C$ & Book $C^{*}$ \\
\hline Book 1 & 4.1 & -0.473466 & -1.941211 & 0.654654 & 2.68408 & 0.93325653 & 3.826352 \\
\hline Book 2 & 4.7 & -0.198223 & -0.931648 & 0.407804 & 0.407804 & 0.78931804 & 3.709795 \\
\hline Total & 8.8 & & -2.872869 & & 4.600758 & & 7.536147 \\
\hline Total/indicator & & & -0.326461 & & 0.522813 & & 0.85638 \\
\hline
\end{tabular}

\subsection{Trend Processing After Regional Sensor Data Fusion}

About the providing of learning service environment of library to readers, when the real-time attribute value of a specific area is obtained, we need whether this value can truly describe the indoor environment change. The moving average method is adopted here. Moving average method, with a time sequence $y_{1}$, $y_{2} \ldots y_{t}, \ldots$ Then, the average of $\mathrm{N}$ Numbers can be calculated one by one according to the order of data, and the moving average can be obtained:

$M_{t}^{(1)}=\frac{y_{t}+y_{t-1}+\cdots+y_{t-M+1}}{N}=M_{t-1}^{(1)}+\frac{y_{t}-y_{t-N}}{N}, \quad t \geq \mathrm{N}$

where $M_{t}^{(1)}$ is the first moving average of the t-period; $y_{t}$ is the observed value of the $\mathrm{t}$ period; $\mathrm{N}$ is the number of moving averages, that is, the number of observations used by each moving average.

This formula indicates that when time $\mathrm{t}$ moves forward to the next moment, a new data is added, the earliest data is removed, and a new average is finally obtained. Because it continues to absorb the old and absorb the new and move forward one time point after another, it is called moving average method. Because the data can be smoothed by the moving average method, it can eliminate the influence of periodic and irregular changes too. It makes the long-term trend of the data appears; it can be used for forecasting. Its prediction formula is:

$\hat{y}_{t+1}=M_{t}^{(1)}$

That is, take the average value of the first moving of the $t$ period as the predicted value of the $t+1$ period. For example, indoor temperature as shown in Fig. 4, after moving average method of value was compared with the original values as shown in Fig. 5, can be seen clearly from the table, after processing the original two peaks has become smooth and that they will have much impact on the whole, but also will not mislead the system's response to events, have played an important role in forecasting.

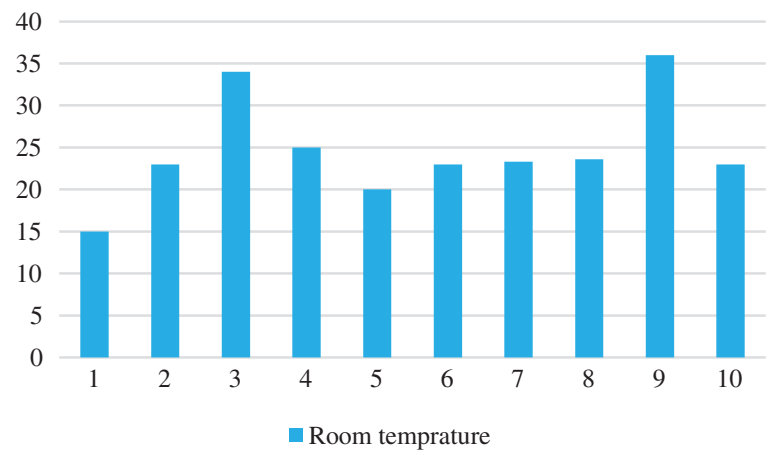

Figure 4: Actual temperature curve of the room 


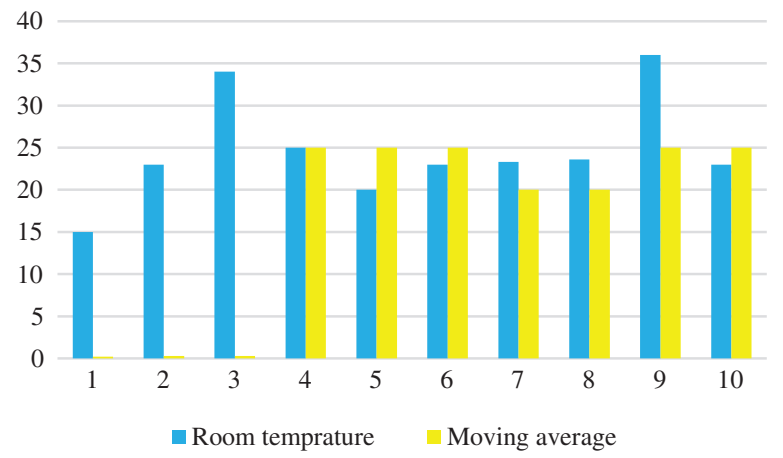

Figure 5: Comparison of renderings

Fig. 5 shows the prediction graph given by applying the moving average method to verify the feasibility of the method when the value of sensors in the home keeps rising and the temperature value of room a keep rising.

\section{Conclusions}

In order to better provide personalized mobile reading services for readers under the mobile environment, this paper applies the item recommendation based on situational awareness to the field of digital library and proposes a situational awareness recommendation method based on collaborative filtering. This method measures readers' situational awareness through "situational entropy" and calculates the corresponding weight values of different situational attributes in information recommendation. On this basis, combining with the traditional collaborative filtering technology, it provides readers with personalized reading recommendation services in line with the current situation. Experimental results show that the method is superior to the traditional recommendation method based on collaborative filtering, can well predict the reader perception properties on the situation, and identify the different role in the different situation for information recommendation, which based on the current situation in mobile environment information, accurately provide personalized mobile reading service for readers. The research of this paper makes beneficial exploratory research on the personalized information recommendation service of digital library based on context perception in mobile environment. It can be predicted that the mobile reading recommendation service, which centers on readers and provides them with situational awareness, will be a new direction for the development of information service of digital library.

Funding Statement: This work is funded by the National Social Science Fund of China (No. 19BTQ045). Haixu Xi received the grant and the URLs to sponsors' websites is http://fund.cssn.cn/skjj/.

Conflicts of Interest: The authors declare that they have no conflicts of interest to report regarding the present study.

\section{References}

[1] R. A. Baryshev, S. V. Verkhovets and O. I. Babina, "The smart library project: Development of information and library services for educational and scientific activity," The Electronic Library, vol. 36, no. 3, pp. 535-549, 2018.

[2] N. A. R. L. Netto and C. L. T. Borges, "Enhancing the situational awareness of voltage security region via probabilistic reliability evaluation," International Transactions on Electrical Energy Systems, vol. 30, no. 1, pp. e12150, 2020. 
[3] C. P. Ge, W. Susilo, J. Baek, Z. Liu, J. Xia et al., "Revocable attribute-based encryption with data integrity in clouds," IEEE Transactions on Dependable and Secure Computing, vol. 21, no. 3, pp. 1-12, 2021.

[4] Y. Ren, Y. Leng, J. Qi, K. S. Pradip, J. Wang et al., "Multiple cloud storage mechanism based on blockchain in smart homes," Future Generation Computer Systems, vol. 115, no. 3, pp. 304-313, 2021.

[5] A. Kissinger-Knox, P. Aragon and M. Mizrahi, "Does Non-moral ignorance exculpate? situational awareness and attributions of blame and forgiveness," Acta Analytica, vol. 33, no. 2, pp. 161-179, 2018.

[6] N. Pogrebnyakov and E. Maldonado, "Didn't roger that: Social media message complexity and situational awareness of emergency responders," International Journal of Information Management, vol. 40, pp. 166$174,2018$.

[7] C. Ge, Z. Liu, J. Xia and L. Fang, "Revocable identity-based broadcast proxy re-encryption for data sharing in clouds," IEEE Transactions on Dependable and Secure Computing, vol. 18, no. 3, pp. 1214-1226, 2021.

[8] G. Cao, M. Liang and X. Li, "How to make the library smart? the conceptualization of the smart library," The Electronic Library, vol. 36, no. 5, pp. 811-825, 2018.

[9] Y. J. Ren, Y. Leng, Y. Cheng and J. Wang, "Secure data storage based on blockchain and coding in edge computing," Mathematical Biosciences and Engineering, vol. 16, no. 4, pp. 1874-1892, 2019.

[10] J. Wang, "Readers' privacy protection technology under the smart library mode," Libraly Journal, vol. 36, no. 9, pp. 82-88, 2017.

[11] J. Lim, "Additional helmet and pack loading reduce situational awareness during the establishment of marksmanship posture," Ergonomics, vol. 60, no. 6, pp. 824-836, 2017.

[12] Y. Ren, F. Zhu, K. S. Pradip, T. Wang, J. Wang et al., "Data query mechanism based on hash computing power of blockchain in internet of things," Sensors, vol. 20, no. 1, pp. 1-22, 2020.

[13] G. Xu, X. Li, L. Jiao, W. Wang, A. Liu et al., "BAGKD: A batch authentication and group key distribution protocol for VANETs," IEEE Communications Magazine, vol. 58, no. 7, pp. 35-41, 2020.

[14] Y. Li, G. Huang, C. Wang and Y. Li, "Analysis framework of network security situational awareness and comparison of implementation methods," EURASIP Journal on Wireless Communications and Networking, vol. 2019, no. 1-8, pp. 205, 2019.

[15] Y. J. Ren, J. Qi, Y. Cheng, J. Wang and A. Osama, "Digital continuity guarantee approach of electronic record based on data quality theory," Computers Materials \& Continua, vol. 63, no. 3, pp. 1471-1483, 2020.

[16] E. D. Rosenman, "A Simulation-based approach to measuring team situational awareness in emergency medicine: A multicenter, observational study," Academic Emergency Medicine, vol. 25, no. 2, pp. 196-204, 2018.

[17] L. Russell, R. Goubran, F. Kwamena and F. Knoefel, "Agile IoT for critical infrastructure resilience: Cross-modal sensing as part of a situational awareness approach," IEEE Internet of Things Journal, vol. 5, no. 6, pp. 44544465, 2018.

[18] T. R. Gonçalves, "Assessment of brazilian monovarietal olive oil in two different package systems by using data fusion and chemometrics," Food Analytical Methods, vol. 13, no. 1, pp. 86-96, 2020.

[19] L. Fang, Y. Li, X. Yun, Z. Wen, S. Ji et al., "THP: A novel authentication scheme to prevent multiple attacks in SDN-based IoT network," IEEE Internet of Things Journal, vol. 7, no. 7, pp. 5745-5759, 2020.

[20] Z. Wang, "Data fusion in data scarce areas using a back-propagation artificial neural network model: A case study of the South China Sea," Frontiers of Earth Science, vol. 12, no. 2, pp. 280-298, 2018.

[21] T. Li, Y. Ren and J. Xia, "Blockchain queuing model with non-preemptive limited-priority," Intelligent Automation \& Soft Computing, vol. 26, no. 5, pp. 1111-1122, 2020.

[22] J. Hu, G. Hu, J. Cai, L. Xu and Q. Wang, "Hospital bed allocation strategy based on queuing theory during the COVID-19 Epidemic," Computers Materials \& Continua, vol. 66, no. 1, pp. 793-803, 2021.

[23] A. Campbell and T. Harlan, "Using real-time data to warn nurses of medication administration errors using a nurse situational awareness dashboard," Studies in Health Technology and Informatics, vol. 250, no. 5, pp. 140-141, 2018.

[24] C. Ge, W. Susilo, Z. Liu, J. Xia, P. Szalachowski et al., "Secure keyword search and data sharing mechanism for cloud computing," IEEE Transactions on Dependable and Secure Computing, vol. 20, no. 8, pp. 1-12, 2020. 
[25] Y. Ren, J. Qi, Y. Liu, J. Wang and G. Kim, "Integrity verification mechanism of sensor data based on bilinear map accumulator," ACM transactions on Internet Technology, vol. 21, no. 1, pp. 1-19, 2021.

[26] M. K. Al-Sharman, B. J. Emran, M. A. Jaradat, H. Najjaran, R. Al-Husari et al., "Precision landing using an adaptive fuzzy multi-sensor data fusion architecture," Applied Soft Computing, vol. 69, no. 3, pp. 149-164, 2018.

[27] Y. J. Ren, F. Zhu, J. Wang, P. Sharma and U. Ghosh, "Novel vote scheme for decision-making feedback based on blockchain in internet of vehicles," IEEE Transactions on Intelligent Transportation Systems, vol. 21, no. 8, pp. $1-10,2021$.

[28] J. Hu, L. Zhang, J. Li and Q. Wang, "A new mixed clustering-based method to analyze the gait of children with cerebral palsy," Computers Materials \& Continua, vol. 66, no. 2, pp. 1551-1562, 2021.

[29] D. Xi, "Improvement of mammographic mass classification performance using an intelligent data fusion method," Journal of Medical Imaging and Health Informatics, vol. 8, no. 2, pp. 275-283, 2018.

[30] C. P. Ge, W. Susilo, J. Baek, Z. Liu, J. Y. Xia et al., "A verifiable and fair attribute-based proxy re-encryption scheme for data sharing in clouds," IEEE Transactions on Dependable and Secure Computing, vol. 21, no. 7, pp. 1-12, 2021.

[31] M. Beggas, "Towards a model of context-aware infrastructure in mobile ubiquitous computing," Journal of Fundamental and Applied Sciences, vol. 3, no. 1, pp. 47-57, 2015.

[32] T. Zhao, J. Fu, Y. Wang and B. Zhang, "An underwater measurement and control network centralized data fusion localization algorithm based on Chan-algorithm method," The Journal of the Acoustical Society of America, vol. 142, no. 4, pp. 2732, 2017.

[33] L. Fang, M. Li, Z. Liu, C. Lin, S. Ji et al., "A secure and authenticated mobile payment protocol against off-site attack strategy," IEEE Transactions on Dependable and Secure Computing, vol. 21, no. 8, pp. 77-90, 2021.

[34] A. R. Di Rosa, F. Leone, C. Scattareggia and V. Chiofalo, "Botanical origin identification of Sicilian honeys based on artificial senses and multi-sensor data fusion," European Food Research and Technology, vol. 244, no. 1, pp. $117-125,2018$.

[35] A. Makni, A. Y. Kibangou and H. Fourati, "Data fusion-based descriptor approach for attitude estimation under accelerated maneuvers," Asian Journal of Control, vol. 21, no. 4, pp. 1433-1442, 2019. 